\title{
A new inversion algorithm for backscatter ionogram and its experimental validation
}

\author{
J. J. Zhao, C. Zhou, G. B. Yang, C. H. Jiang, S. S. Chang, P. Zhu, X. D. Gu, B. B. Ni, and Z. Y. Zhao \\ Ionosphere Laboratory, School of Electronic Information, Wuhan University, Wuhan, Hubei 430072, China \\ Correspondence to: C. Zhou (chen.zhou.whu@gmail.com) \\ Received: 2 November 2013 - Revised: 11 March 2014 - Accepted: 11 March 2014 - Published: 28 April 2014
}

\begin{abstract}
Oblique backscatter sounding is a powerful tool for detecting and monitoring the ionosphere continuously at a remote distance. High-frequency (HF) backscatter ionograms provide the amplitudes of backscatter signals with respect to group path or time delay against operating frequency. Application of inversion algorithm to a backscatter ionogram can extract useful information regarding the ionospheric electron density along the propagation paths. The present study proposes a new inversion algorithm on basis of simulated annealing method to acquire the leading edge of sweep-frequency ionogram, which is subsequently validated by ionospheric vertical sounding data. Quantitative comparisons between the vertical sounding measurements and the inversion results obtained from oblique backscatter sounding indicate that the new algorithm enables us to overcome the instability issue that traditional inversion algorithm faces and output reliable information of ionospheric inversion with satisfactory efficiency, thus providing a robust alternative for ionospheric detection based on oblique backscatter ionograms especially when the ionosphere is calm with slow changes.
\end{abstract}

Keywords. Radio science (ionospheric propagation)

\section{Introduction}

The oblique backscatter sounding is a powerful tool for detecting the ionosphere, and it can be used to monitor the ionosphere continuously at a remote distance (Croft, 1972). When a high-frequency (HF) signal is emitted with an angle with respect to the horizon, it can be reflected by the ionosphere, and then scattered by the terrestrial surface. In this case, weak scattered echoes can be received at the same location of the transmitter. The ionospheric parameter inversion of backscatter ionograms is an important means to study the ionosphere and to improve the performance of sky wave over the horizon radar. There are two typical kinds of backscatter ionograms. One consists of transmitting a signal at a fixed azimuth and operating frequency by scanning in elevation (Saillant et al., 2003). The other is obtained by scanning in frequency when available radars are not able to scan in elevation (Coleman, 1997), which is named sweep frequency backscatter ionogram (SFBI). For the latter, the most important characteristic of backscatter signals is timedelay focusing, which often causes the backscatter echoes to occur abruptly so that the time delay of the leading edge can be identified and measured with confidence. Backscatter ionogram inversion aims to extract the information carried by oblique backscatter sounding signals to acquire ionospheric parameters (Fridman and Fridman, 1994). Although the backscatter ionogram contains useful information for inversion purposes, it is difficult to utilize the whole ionogram, since refraction by the ionosphere, Earth's magnetic field, radio wave polarization, and absorption and scattering of the ground surface combines together to contribute to the profiles of backscatter ionogram. However, since the time of backscatter signal propagation is not considerably affected by any factor other than the electron density distribution in the ionosphere, sweep-frequency ionogram has a steep forward front, which can be easily identified as a leading edge for use to invert the distribution of ionospheric electron density from backscatter ionogram.

A variety of approaches aiming to resolve the inversion problem have been proposed. For instance, Rao (1974) presented an iterative method for the derivation of quasiparabolic ionospheric layer parameters (the critical frequency of the ionized layer, the maximum height, and halfthickness) from three data points on the leading edge of a 
sweep-frequency sky-wave backscatter ionogram. Bertel et al. (1987) found that this method with three data points appeared to be unstable in the iterative calculation, and consequently increased the number of data points to 15 or more to improve the stability. Norman (2003) further improved this method by using the quasi-parabolic segment (QPS) model derived by Dyson and Bennett (1988). The QPS model uses five QPSs to represent the E, F1, and F2 ionospheric layers. All the above methods are restricted to real-time data because the inverse problem is mathematically ill-posed. Input data are strictly required to be collected from the leading edge besides the initial ionosphere model parameters. Also, a large number of matrixes need to be solved during the iterative course. As a consequence, the computation process is vulnerable to becoming unstable. Fridman and Fridman (1994) developed a new method that uses the sweep-frequency ionogram in combination with the vertical ionogram to invert the simulated ionogram. Fridman (1998) tested that algorithm by applying it to real-time data, and reconstructed a 3-D ionospheric electron density. Ruelle and Landeau (1994) also stabilized the inverse problem by using elevation angle data and demonstrated through a Bayesian approach. Landeau et al. (1997) made a further improvement by applying the inversion of elevation-scan backscatter sounding data. Recently, Norman and Dyson (2006) validated the iterative method on real data obtained by elevation scan, and Benito et al. (2008) presented the method that was stable and fast enough for a real-time application.

In the present study, a new method is developed to obtain the distribution of ionospheric electron density from the leading edge of sweep-frequency backscatter ionograms. This technique utilizes the simulated annealing method to obtain the global optimum solver, which is found to be stable and fast. This method has been validated on real data collected by the Wuhan Ionospheric Oblique Backscattering Sounding System (WIOBSS) (Chen et al., 2007; Zhou et al., 2010). To validate the inversion method proposed in this study, we carry out three representative experiments with the antenna directing southward, westward, and northwestward, respectively. Simultaneous vertical sounding data are collected to compare with the backscatter ionogram inversion results. A general description of the inversion algorithm with the analytic ionospheric model is given in Sect. 2. Section 3 presents the details of the three experiment arrangements and illustrates the inversion results as well. We discuss the results in Sect. 4 , followed by the major conclusions summarized in Sect. 5.

\section{Details of inversion algorithm}

\subsection{Ionospheric model}

Before any inversion process, an ionospheric model is required. Here we adopt the quasi-parabolic (QP) layer model defined by Croft and Hoogasian (1968), as given below,

$N_{\mathrm{e}}(r)=\left\{\begin{array}{l}N_{\mathrm{m}}\left[1-\left(\frac{r-r_{\mathrm{m}}}{y_{\mathrm{m}}}\right)^{2}\left(\frac{r_{\mathrm{b}}}{r}\right)^{2}\right], r_{\mathrm{b}}<r<r_{\mathrm{m}}\left(\frac{r_{\mathrm{b}}}{r_{\mathrm{b}}-y_{\mathrm{m}}}\right) \\ 0, \text { otherwise }\end{array}\right.$,

where $N_{\mathrm{e}}$ is the electron density at a radial distance $r$ from the Earth's center, $N_{\mathrm{m}}$ is the maximum electron density at the radial height $r_{\mathrm{m}}, r_{\mathrm{b}}$ is the radial base height of the ionospheric layer and $y_{\mathrm{m}}$ is the semi-layer thickness. For HF radio waves propagating in the ionosphere, the group path $P^{\prime}$ and ground distance $D$ for a single QP layer are given by

$$
\begin{aligned}
P^{\prime} & =2\left\{r_{\mathrm{b}} \sin \gamma-r_{0} \sin \beta+\frac{1}{A}\left[-r_{\mathrm{b}} \sin \gamma\right.\right. \\
& \left.\left.-\frac{B}{4 \sqrt{A}} \ln \frac{B^{2}-4 A C}{\left(2 A r_{\mathrm{b}}+B+2 r_{\mathrm{b}} \sqrt{A} \sin \gamma\right)^{2}}\right]\right\}
\end{aligned}
$$

$D=$

$$
2 r_{0}\left\{(\gamma-\beta)-\frac{r_{0} \cos \beta}{2 \sqrt{C}} \ln \frac{B^{2}-4 A C}{4 C\left(\sin \gamma+\frac{\sqrt{C}}{r_{\mathrm{b}}}+\frac{B}{2 \sqrt{C}}\right)^{2}}\right\}
$$

with $A=1-\frac{1}{F^{2}}+\left(\frac{r_{\mathrm{b}}}{F y_{\mathrm{m}}}\right)^{2}, B=-\frac{2 r_{\mathrm{m}} r_{\mathrm{b}}^{2}}{F^{2} y_{\mathrm{m}}^{2}}, C=\left(\frac{r_{\mathrm{b}} r_{\mathrm{m}}}{F y_{\mathrm{m}}}\right)^{2}-$ $r_{0}^{2} \cos ^{2} \beta, F=f / f_{\mathrm{c}}, r_{0}$ is the earth radius, $f_{\mathrm{c}}$ is the critical frequency, $\beta$ is the elevation angle, and $\gamma$ is the angle of the ray at the bottom of the ionosphere.

\subsection{Algorithm}

We need to determine the elevation angle corresponding to each minimum time delay on the leading edge of the sweepfrequency ionogram. For a given frequency and a set of layer parameters, the relationship between group path and elevation angle is shown in Fig. 1. Figure 1 demonstrates a typical focusing effect of HF radio wave propagation in the ionosphere. The focusing effect determines that the trend of group distance in function of the elevation angle has a minimum value and enhances the power in the leading edge of the backscatter ionogram. This characteristic has been studied by many authors (Georges and Stephenson, 1969; Croft, 1967, 1972; Ruelle and Landeau, 1994; Landeau et al., 1997). At the beginning, group path $P^{\prime}$ decreases while $\beta$ increases. The minimum group delay $P_{\min }^{\prime}$ is reached when $\beta=\beta_{\min }$. When $\beta>\beta_{\min }, P^{\prime}$ increases monotonically with $\beta$. Based on Fig. 1, elevation angle can be solved numerically. Let $\beta_{1}=0.99 \beta_{v}$ in which $\beta_{v}$ is the angle when the penetration condition occurs, $\beta_{2}=0.95 \beta_{1}$, and $\beta_{3}=0.90 \beta_{1}$; then the group paths $P_{1}^{\prime}, P_{2}^{\prime}, P_{3}^{\prime}$ corresponding to $\beta_{1}, \beta_{2}, \beta_{3}$ are obtained by a ray tracing method. If $P_{1}^{\prime}>P_{2}^{\prime}>P_{3}^{\prime}, \beta_{1}$ and $P_{1}^{\prime}$ are discarded, and the subscripts 2 and 3 are changed to be 1 and 2 , then the new group path $P_{3}^{\prime}$ corresponding to the new elevation $\beta_{3}=0.95 \beta_{2}$ is calculated. If $P_{1}^{\prime}<P_{2}^{\prime}<P_{3}^{\prime}, \beta_{3}$ and 


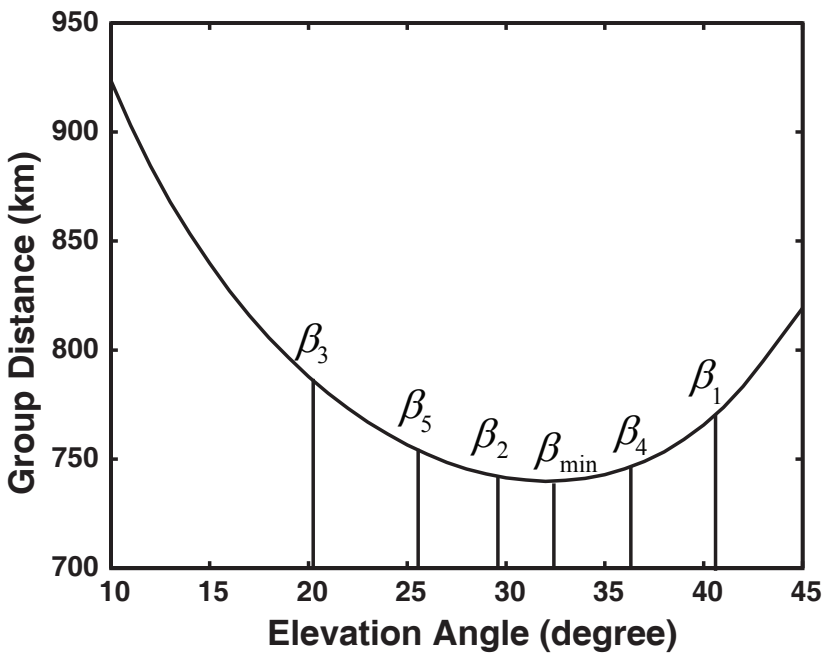

Fig. 1. Variation of group distance with elevation angle for a certain transmitted frequency, where $\beta_{1}, \beta_{2}, \beta_{3}, \beta_{4}, \beta_{5}$, means different elevation angle and $\beta_{\min }$ is the elevation angle corresponding to minimum group distance.

$P_{3}^{\prime}$ are discarded, and the subscripts 1 and 2 are changed to be 2 and 3 , then the new group path $P_{1}^{\prime}$ corresponding to the new elevation $\beta_{1}=1.05 \beta_{2}$ is calculated. By taking into account the above various conditions, the computation process is repeated until the situation that $P_{1}^{\prime}>P_{2}^{\prime}$ and $P_{3}^{\prime}>P_{2}^{\prime}$ is reached, as seen in Fig. 1. Subsequently, the following steps are followed in order to obtain the minimum group delay within a tolerable error $\delta P$.

1. Choose $\beta_{4} \in\left[\beta_{1}, \beta_{2}\right]$ and $\beta_{5} \in\left[\beta_{2}, \beta_{3}\right]$, and the corresponding group paths $P_{4}^{\prime}$ and $P_{5}^{\prime}$ are calculated by ray tracing.

2. If $\left|P_{5}^{\prime}-P_{2}^{\prime}\right|>\delta P$, turn to step 3. If not, take the minimum of $P_{2}^{\prime}, P_{4}^{\prime}, P_{5}^{\prime}$ as the minimum group path $P_{\min }^{\prime}$, and terminate the process.

3. If $P_{5}^{\prime}>P_{2}^{\prime}$, turn to step 4 . If not, as the two ranges involving $P_{3}^{\prime}>P_{5}^{\prime}$ and $P_{2}^{\prime}>P_{5}^{\prime}$ are most concerned, replace $\beta_{1}, \beta_{2}, P_{1}^{\prime}, P_{2}^{\prime}$ with $\beta_{2}, \beta_{5}, P_{2}^{\prime}, P_{5}^{\prime}$, and the process turns to step 1 from the beginning.

4. If $\left|P_{4}^{\prime}-P_{2}^{\prime}\right|>\delta P$, turn to step 5. If not, take the smaller of $P_{2}^{\prime}, P_{4}^{\prime}$ as $P_{\min }^{\prime}$, and terminate the process.

5. If $P_{4}^{\prime}>P_{2}^{\prime}$, the two ranges involving $P_{5}^{\prime}>P_{2}^{\prime}$ and $P_{4}^{\prime}>P_{2}^{\prime}$ are most concerned. Replace $\beta_{2}, \beta_{3}, P_{2}^{\prime}, P_{3}^{\prime}$ with $\beta_{4}, \beta_{5}, P_{4}^{\prime}, P_{5}^{\prime}$, and the process turns to step 1 from the beginning. If not, the two ranges involving $P_{2}^{\prime}>P_{4}^{\prime}$ and $P_{1}^{\prime}>P_{4}^{\prime}$ are most concerned. Replace $\beta_{2}, \beta_{3}, P_{2}^{\prime}, P_{3}^{\prime}$ with $\beta_{4}, \beta_{2}, P_{4}^{\prime}, P_{2}^{\prime}$, and the process turns to step 1 from the beginning.

No matter whether there is an analytic solution, the above method is robust and efficient. While a number of elevation angles corresponding to different frequencies need to be identified, the previous $\beta_{\min }$ can be used as the initial value of the searching process to improve computational efficiency.

The purpose of the inversion method is to acquire the ionosphere parameters from the leading edge. Following the work of Benito et al. (2008), the new a posteriori probability density $\sigma_{p}$ over the parameter space $\left(f_{\mathrm{c}}, h_{\mathrm{m}}, y_{\mathrm{m}}\right)$ is given by

$$
\begin{aligned}
& \sigma_{p}\left(f_{\mathrm{c} F}, h_{\mathrm{m} F}, y_{\mathrm{m} F}\right)= \\
& \quad-\prod_{i=1}^{N} \exp \left(-\frac{1}{2}\left(\frac{P_{i}^{\prime}\left(f_{\mathrm{c}}, h_{\mathrm{m}}, y_{\mathrm{m}}\right)-P_{r i}^{\prime}}{\delta P_{i}^{\prime}}\right)^{2}\right), N \geq 3,
\end{aligned}
$$

where $f_{\mathrm{c}}$ and $y_{\mathrm{m}}$ have been defined in the previous text; $h_{\mathrm{m}}$ is the real height between the maximum electron density point and the ground. Consequently, $h_{\mathrm{m}}=r_{\mathrm{m}}-r_{0} ; N$ cannot be large. Otherwise, the absolute value of $\sigma_{p}$ is too small to be identified during the searching process. $P_{r i}^{\prime}, i=1,2, \ldots, N$ are the group paths on the leading edge corresponding to operating frequencies $f_{i}, i=1,2, \ldots, N$ on the real ionogram, and $P_{i}^{\prime}\left(f_{\mathrm{c}}, h_{\mathrm{m}}, y_{\mathrm{m}}\right), i=1,2, \ldots, N$ are the group paths calculated at the same frequencies by using a QP model, where the parameters are taken from the parameters space; $\delta P_{i}^{\prime}$ represents the variances of the measurements errors over $P_{r i}^{\prime}$, which are assumed to be Gaussian and uncorrelated. Each time before calculating $\sigma_{p}$, the elevation angle is required. This method evaluates the a posteriori probability density $\sigma_{p}$ over the parameter space to locate the minimum of this function. The simulated annealing (SA) (Kirkpatricketal et al., 1983) algorithm, a geophysical optimization algorithm, is carefully implemented to reduce the computation time. SA provides a good approximation to the global optimum of a given function in a large search space. By using this optimization algorithm, the computation time can reduce to within a few minutes. When the minimum of $\sigma_{p}$ is found, the final solution of the layer parameters is obtained.

\section{Experimental validations of the inversion method}

\subsection{Experiment facilities}

The Wuhan Ionospheric Oblique Backscattering Sounding System (WIOBSS), which is developed by the Ionosphere Laboratory of Wuhan University, is a low-power and monostatic HF sky-wave radar aimed at ionospheric research and HF channel management. The principal specifications of WIOBSS are given in Table 1. The detailed description of WIOBSS is given in Chen et al. (2007) and Zhou et al. (2010). To test and verify the accuracy of the inversion method, an ionospheric vertical incident sounder was located in the coverage of the backscatter radar. This vertical sounder is basically same as the WIOBSS but with cobra antenna for transmitter and an orthogonal cobra antenna for receiver. 


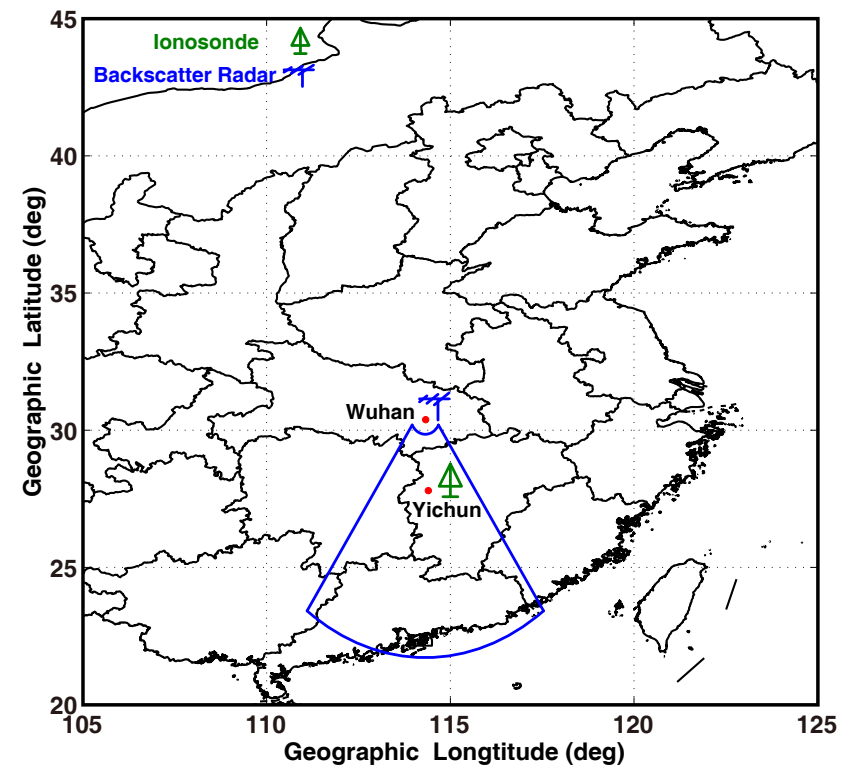

Fig. 2. Map showing the location of the WIOBSS at Wuhan and the location of the vertical ionosonde at Yichun for the experiment on 25 and 26 August 2010. The sector denotes the coverage of WIOBSS.

\subsection{Experimental results}

\subsubsection{Experimental test I}

The inversion method proposed in this study is firstly tested using the real data recorded from 10:30 to 12:30 local time (LT) on 25 August 2010 and from 10:30 to 20:30 LT on 26 August 2010. WIOBSS recorded the data and produced backscatter ionogram every $30 \mathrm{~min}$. The vertical sounder operated simultaneously and recorded the data every $5 \mathrm{~min}$. The log-periodic antenna of WIOBSS was located at Wuhan $\left(30.5^{\circ} \mathrm{N}, 114.3^{\circ} \mathrm{E}\right)$, pointing southward. The vertical ionosonde was located at Yichun $\left(27.7^{\circ} \mathrm{N}, 114.3^{\circ} \mathrm{E}\right)$, about $316 \mathrm{~km}$ south of Wuhan. Figure 2 is a schematic map of experiment I, in which the sector indicates the coverage of WIOBSS.

Figure 3a demonstrates the raw backscatter ionogram recorded by WIOBSS at 12:00 LT on 26 August 2010. The red curves at the low frequency band are the vertical and nearly vertical echoes due to the energy transmitted from the side lobe of the log-periodic antenna. The backscatter echoes occurred at most frequencies and formed a leading edge due to focusing effects. All the data points on the leading edge of the backscatter ionogram have been used as input for the inversion. We divide the leading edge into several groups that do not overlap with each other according to the operating frequency. Since the operating frequency difference in one group was small, we assume that the frequencies in each group encounter the same state of the ionosphere. The inversion algorithm is applied to each frequency group
Table 1. WIOBSS main specifications.

\begin{tabular}{ll}
\hline Item & Specification \\
\hline Operating Frequency & $6-30 \mathrm{MHz}$ \\
Sweep-frequency step & $200 \mathrm{kHz}$ \\
Peak Power & $200 \mathrm{~W}$ \\
Antenna & Log-periodic antenna \\
\hline
\end{tabular}
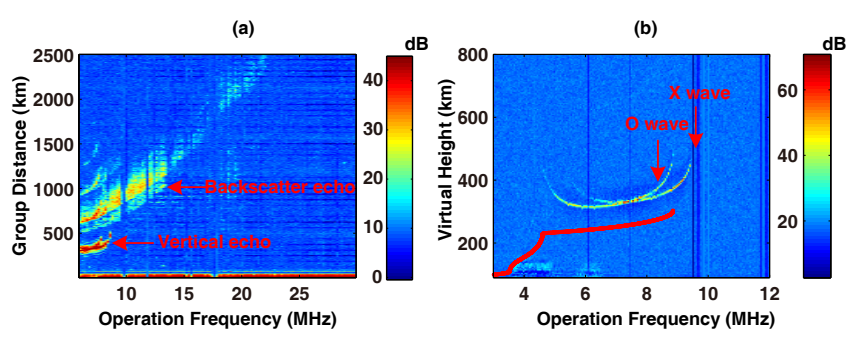

(c)

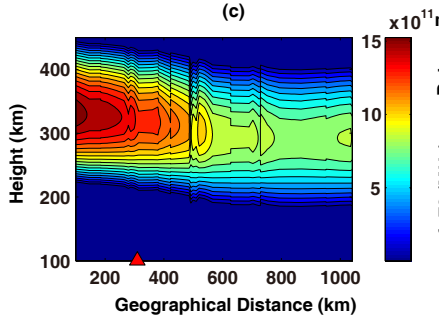

$\times 10^{11} \mathrm{~m}^{-3} \quad$ (d)

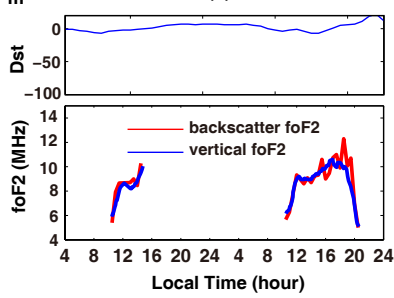

Fig. 3. (a) Backscatter ionogram recorded by the Wuhan WIOBSS at 12:00 LT on 26 August 2010. (b) Vertical ionogram recorded by the Yichun ionosonde at 12:00 LT on 26 August 2010. The red curve is the electron density profile inverted using POLAN method. (c) 2$\mathrm{D}$ distribution of inverted ionospheric electron density as a function of geographical ground distance and height in the direction of antenna main beam. The red triangle indicates the location of the vertical ionosonde at Yichun. (d) Comparison of $f o \mathrm{~F} 2$ obtained from the vertical ionosonde (blue curves) and foF2 inverted using the backscatter ionogram at a ground distance of $316 \mathrm{~km}$ (red curves).

independently. We also assume that different portions of the leading edge represent different conditions of the ionospheric state that correspond to different ground ranges. For instance, the leading edge is divided into 20 parts. Each part has three data points, and the total number of data points on the leading edge is 60. After the entire leading edge is inverted, we use Eq. (3) to compute all the ground ranges and select the parameters corresponding to the distance of $316 \mathrm{~km}$ for a quantitative comparison with the vertical data of the ionospheric obtained by the ionosonde located at Yichun. To acquire this ground range reliably close to $316 \mathrm{~km}$, we have arranged the interval of data points on the leading edge to be necessarily small.

Figure $3 \mathrm{~b}$ demonstrates the vertical ionogram recorded simultaneously at Yichun at 12:00 LT on 26 August 2010. The two color-coded curves correspond to the O-mode and Xmode waves respectively. The raw ionogram is automatically scaled by using the method similar to Ding et al. (2007). The 
red curve in Fig. $3 b$ shows the electron density profile below the F2 peak height at Yichun at 12:00 LT on 26 August 2010 inverted by the POLAN method (Titheridge, 1985). Figure 3c illustrates the distribution of electron density in the direction of antenna main beam inverted from the ionogram shown in Fig. 3a by using the method introduced in Sect. 2. The red triangle indicates the location of vertical ionosonde at Yichun. Detailed comparison of $f o \mathrm{~F} 2$ obtained by the vertical ionosonde (blue curves) at the ground distance of $316 \mathrm{~km}$ to WIOBBS and $f o F 2$ inverted using the backscatter ionograms and the proposed inversion method (red curves) is illustrated in the bottom panel of Fig. $3 \mathrm{~d}$ as a function of time. The top panel of Fig. 3d illustrates the Dst value during the experiment, indicating that the geomagnetic activity is quiet. For the experiment on 25 August, the inversion results using the backscatter ionogram at Wuhan show an excellent agreement with the localized vertical ionogram results at Yichun, with the maximum relative difference of $11.5 \%$ and the absolute mean relative difference of $7.5 \%$. For the experiment on 26 August, the inversion results agree favorably with the vertical ionogram results for the first $3 \mathrm{~h}$. Later on, the inverted $f_{o} \mathrm{~F} 2$ becomes fluctuated and tends to exceed the values obtained using the vertical ionogram. For the entire $10 \mathrm{~h}$ period of the 26 August experiment, the maximum relative difference is $24.8 \%$, occurring at $\sim 18: 30 \mathrm{LT}$, and the absolute mean relative difference is $8.27 \%$, larger than that for the 25 August experiment. However, the two foF 2 profiles on 26 August acquired from independent means are still notably similar to each other with good consistency, indicating that our inversion method proposed for backscatter ionograms operates reliably to output reasonable information of the ionosphere.

\subsubsection{Experimental test II}

Another experiment was carried out from 12:00 to 21:00 LT on 24 May 2011. WIOBSS was located at Kunshan $\left(31.1^{\circ} \mathrm{N}\right.$, $\left.120.5^{\circ} \mathrm{E}\right)$ with the antenna pointing northwest. The vertical ionosonde was locate at Taihe $\left(33.2^{\circ} \mathrm{N}, 115.3^{\circ} \mathrm{E}\right)$, with a ground distance of $542 \mathrm{~km}$ to Kunshan. Figure 4 illustrates the map of this experiment. The backscatter ionogram was recorded every $30 \mathrm{~min}$ and the vertical ionogram every $5 \mathrm{~min}$. Figure 5a illustrates the raw backscatter ionogram recorded at 16:15 LT on 24 May 2011. The leading edge of backscatter ionogram illustrated in Fig. 5a is relatively flatter than in Fig. 3a, which indicates that the electron density is thinner than that corresponding to Fig. 3a. Figure 5b illustrates the raw vertical ionogram recorded simultaneously by the ionosonde at Taihe, in which the red curve represents the inverse electron density profile. Figure $5 \mathrm{c}$ presents the inversion results of ionospheric electron density corresponding to Fig. 5a, which are two-dimensional as a function of geographical ground distance and height. Bottom panel of Fig. 5d shows the temporal comparison of inverse $f o \mathrm{~F} 2$ obtained from the vertical ionogram (blue curve) and the

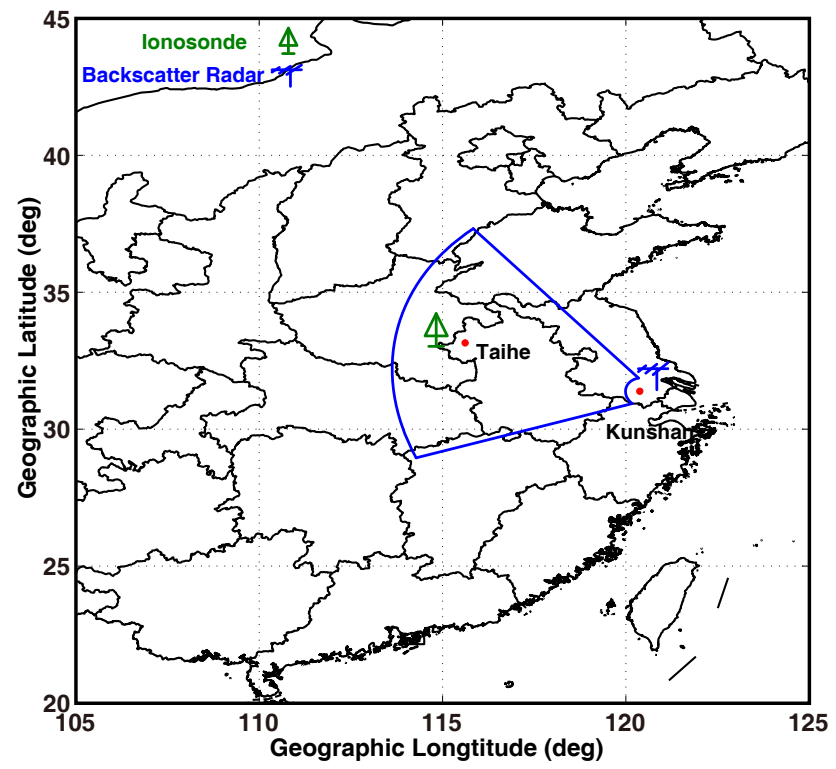

Fig. 4. Map showing the location of the WIOBSS at Kunshan and the location of the vertical ionosonde at Taihe for the experiment on 24 May 2011.

backscatter ionogram (red curve) for the entire experiment period. Top panel of Fig. $5 \mathrm{~d}$ also indicates that the geomagnetic condition is calm during this experiment. Although during the period of 14:15 to 18:45 LT on 24 May 2011 the inversion results using the backscatter ionogram are somehow smaller than those using the vertical ionogram, the agreement is favorable for the other time intervals, and the major feature of $f o \mathrm{~F} 2$ variation with time is consistent between the two profiles obtained independently. Specifically, for this northwest direction experiment with a ground distance of $542 \mathrm{~km}$ between the two facilities, the absolute mean relative difference is $5.16 \%$, with a maximum relative difference of $21.6 \%$ at 18:00 LT. These results again indicate that the inversion method is reliable most of the time, while the associated uncertainty may increase at the sunset period.

\subsubsection{Experimental test III}

The third experiment was conducted from 08:15 to 17:15 LT on 7 November 2012 and from 09:15 to 11:15 LT on 8 November 2012. WIOBSS was located at Kunshan $\left(31.1^{\circ} \mathrm{N}, 120.5^{\circ} \mathrm{E}\right)$, but the antenna pointed westward. The vertical ionosonde was arranged at Wuhan, about $630 \mathrm{~km}$ away from Kunshan. The schematic of this experiment is illustrated in Fig. 6. Figure 7a-d are similar to Figs. 3 and 5, while the recorded background ionogram and vertical ionogram are very different from the above experiments and the inverted 2-D electron density shows a more homogenous variation in the horizontal direction. Top panel of Fig. 7d demonstrates that during the experiment the geomagnetic activity is calm. Bottom panel of Fig. 7d demonstrates again the good consistency between the two temporal variations of 

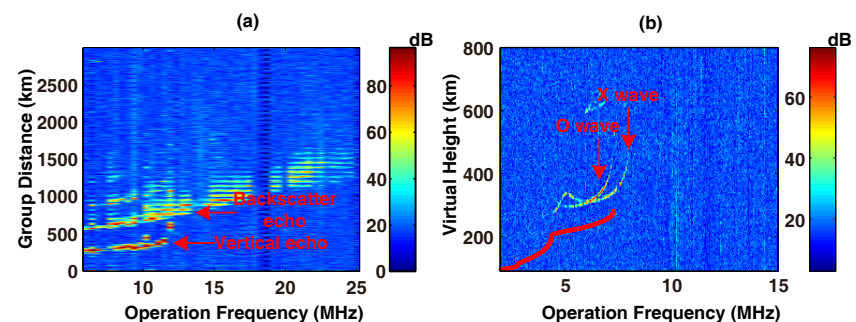

(c)
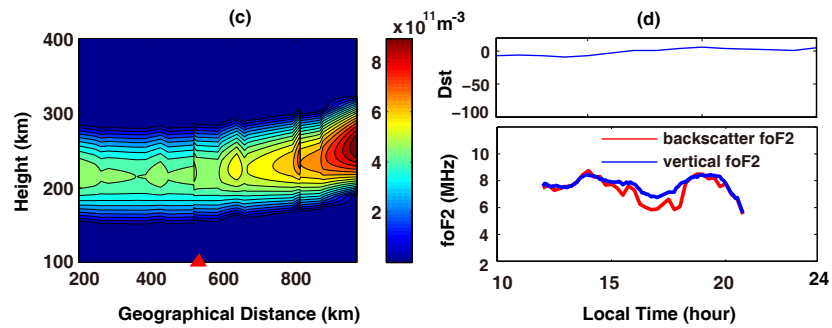

Fig. 5. Same as in Fig. 3, except for the 24 May 2011 experiment illustrated in Fig. 4.

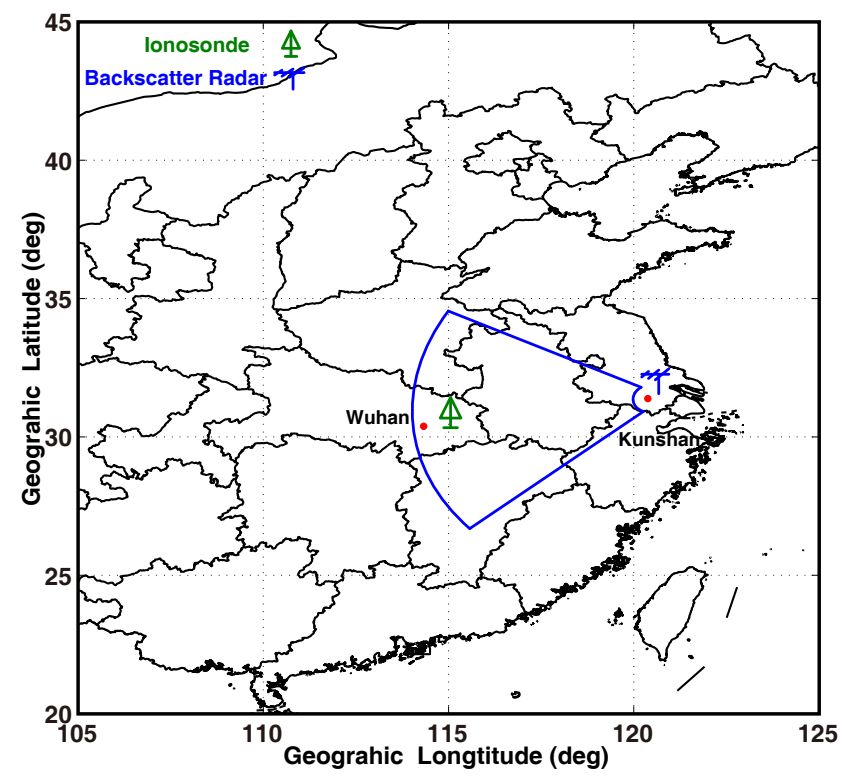

Fig. 6. Map showing the location of the WIOBSS at Kunshan and the location of the vertical ionosonde at Wuhan for the 7 November 2012 experiment.

$f o \mathrm{~F} 2$ for the entire experiment, indicative of the reliability of our proposed method for ionospheric inversion and the applicability of backscatter ionograms for ionospheric sounding. For the experiment on 7 November, the maximum relative difference is $15.7 \%$, which occurs at 13:10 LT, and the absolute mean relative difference is $5.88 \%$. For the experiment on 8 November, the maximum relative difference is $5.95 \%$, which occurs at 10:55 LT, and the absolute mean relative difference is $3.52 \%$.
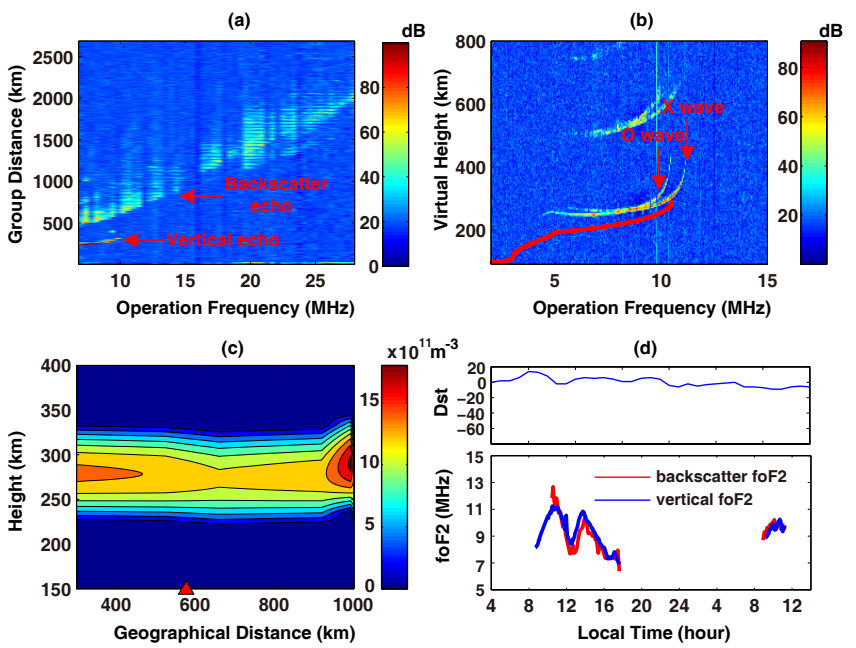

Fig. 7. Same as in Fig. 3, except for the 7 November 2012 experiment illustrated in Fig. 6.

\section{Discussions}

By dealing with all the data, we find that the proposed inversion method can provide reasonable results within a tolerable range of error for either daytime or nighttime data. The root mean square error (RMSE) of the inverse $f o \mathrm{~F} 2$ obtained using the vertical ionogram and the backscatter ionogram independently is $0.4607,0.4178$, and 0.3172 respectively for the three experiments. Although the QP model is adopted by assuming a spherically stratified ionosphere with no external magnetic field, it can be applied to "inhomogeneous" conditions since the principal characteristics of ionospheric electron density dominate the radio wave propagation during the undisturbed condition. Hence, by inversion using the leading edge, we can obtain the information of the ionosphere along the path of sounding waves. The trend of the ionospheric variation in the horizontal direction can be exhibited explicitly. However, we note that errors occurred when the data were collected during the sunset period (18:00-19:30 LT), as shown in Figs. 3d and 5d. During that time, the ionosphere changes rapidly, which can be identified from the vertical sounding directly, and large gradients can exist over the sounding path. The QP ionospheric model is not appropriate under this condition. One group of the leading edge data cannot correspond to the same state of the ionosphere. In Fig. 5d, we could also find inconsistency between $f o \mathrm{~F} 2$ inverted from the vertical ionogram and the backscatter ionogram during 15:00-17:30 LT, 24 May 2011, which is not sunset time interval. We also check the geomagnetic condition during that time, and the Dst index value is 1 , both of which indicate the ionosphere is geomagnetically quiet. We suppose that some local disturbance sources like tropospheric convection or local acoustic/atmospheric gravity waves might affect ionosphere condition. Since the inhomogeneity and/or irregularity of the ionosphere is not included to the ionospheric model 
adopted here, data during sunset need to be analyzed individually. In other words, the adopted method is suitable for the condition when the ionosphere is calm with slow changes.

Different ground ranges can be calculated by dividing the leading edge into several segments. However, we caution that ground distance can vary enormously when the radio wave is reflected by different layers. It is a long-standing issue to identify the echoes from different layers on sweep-frequency backscatter ionograms. While Norman (2003) attempted to invert the three-layer parameters of synthesized ionograms using the QPS model, the information that a realistic ionogram contains is more complicated than a synthesized one. Further work is required to identify and scale reasonably the layer information of realistic ionograms. Another factor that impacts the effectiveness of this method is the auto-scaling of the leading edge and parameter extraction. In this method, leading edge, which contains the ionospheric electron density information, should be scaled accurately (Su et al., 2011; Fridman et al., 2012). Potential improvement can be achieved in practice if auto-scaling is developed more precisely.

The proposed simulated annealing algorithm concerning how to compute elevation angle is easy to carry out, even for a more complicated ionospheric model. It is not necessary to solve equations like $\partial P^{\prime} / \partial \beta=0$. Therefore, during the inversion process, numerical computation time is largely shortened into several minutes, consequently resulting in an applicable efficient inversion method for practical ionospheric detection purposes.

\section{Conclusions}

The present study has proposed a new inversion method based upon simulated annealing algorithm to make full use of backscatter ionograms for ionospheric sounding. By carrying out a number of experiments using the WIOBSS HF ionospheric sounding system and vertical ionosonde, the applicability and the reliability of the inversion method using backscatter ionograms is investigated comprehensively and validated experimentally. By dividing the leading edge data points into several groups, the new inversion method can extract the ionospheric information of different areas along the sounding path and eventually provide useful details of ionospheric parameters. Despite errors that may occur associated with multiple layer echoes or sunset effect, quantitative comparisons between the vertical sounding measurements and the inversion results obtained from oblique backscatter sounding indicate that the new algorithm enables us to overcome the instability issue that traditional inversion algorithm faces and to output reliable information of ionospheric inversion with satisfactory efficiency, thus providing a robust alternative for ionospheric detection based on oblique backscattering sounding, especially when the ionosphere is calm with slow changes.
Acknowledgements. This work was supported by the National Natural Science Foundation of China (NSFC grant no. 41204111). C. Zhou appreciates the support by Wuhan University " 351 Talents Project". We thank the World Data Center for Geomagnetism, Kyoto for providing the Dst data.

Topical Editor K. Hosokawa thanks two anonymous referees for their help in evaluating this paper.

\section{References}

Benito, E., Bourdillon, A., Saillant, S., Rannou, V., and Molinié, J. P.: Inversion of HF backscatter ionograms using elevation scans, J. Atmos. Sol. Terr. Phys., 70, 1935-1948, 2008.

Bertel, L., Cole, D., and Fleury, R.: The inversion of backscatter ionograms, IPS Radio Space Services Technical report IPS-TR88-03, 1987.

Chen, G., Zhao, Z., and Zhang, Y.: Ionospheric Doppler and echo phase measured by the Wuhan Ionospheric Oblique Backscattering Sounding System, Radio Sci., 42, RS4007, doi:10.1029/2006RS003565, 2007.

Coleman, C. J.: On the simulation of backscatter ionograms, J. Atmos. Sol. Terr. Phys., 59, 2089-2099, 1997.

Croft, T. A.: HF radio focusing caused by the electron distribution between ionospheric layers, J. Geophys. Res., 72, 2343-2355, doi:10.1029/JZ072i009p02343, 1967.

Croft, T. A. and Hoogasian, H.: Exact ray calculations in a quasiparabolic ionosphere with no magnetic field, Radio Sci., 3, 6974, 1968.

Croft, T. A.: Sky-wave backscatter: A means for observing our environment at great distances, Rev. Geophys., 10, 73-155, doi:10.1029/RG010i001p00073, 1972.

Ding, H. Z., Ning, B., and Wan, W.: Real-time automatic scaling and analysis of ionospheric ionogram parameters, Chinese J. Geophys., 50, 969-978, 2007 (in Chinese).

Dyson, P. L. and Bennett, J. A.: A model of the vertical distribution of the electron concentration in the ionosphere and its application to oblique propagation studies, J. Atmos. Sol. Terr. Phys., 50, 251-262, 1988.

Fridman, O. V. and Fridman, S. V.: A method of determine horizontal structure of the ionosphere from backscatter ionograms, J. Atmos. Sol. Terr. Phys., 56, 115-131, 1994.

Fridman, S. V.: Reconstruction of a three-dimensional ionosphere from backscatter and vertical ionograms measured by over-the-horizon radar, Radio Sci., 33, 1159-1171, doi:10.1029/98RS00477, 1998.

Fridman, S. V., Nickisch, L. J., and Hausman, M.: Inversion of backscatter ionograms and TEC data for over-the-horizon radar, Radio Sci., 47, RS0L10, doi:10.1029/2011RS004932, 2012.

Georges, T. M. and Stephenson, J. J.: HF Radar Signatures of Traveling Ionospheric Irregularities, 3D Ray-Tracing Simulation, Radio Sci., 4, 679-696, doi:10.1029/RS004i008p00679, 1969.

Kirkpatrick, S., Gelatt, C. D., and Vecchi, M. P.: Optimization by simulated annealing, Science, 220, 671-680, 1983.

Landeau, T., Gauthier, F., and Ruelle, N.: Further improvements to the inversion of elevation-scan backscatter sounding data, J. Atmos. Sol. Terr. Phys., 59, 125-138, 1997.

Norman, R. J.: Backscatter ionogram inversion, Proceedings of the International Conference on Radar, Australia. IEEE Press, 368374, 2003. 
Norman, R. J. and Dyson, P. L.: HF radar backscatter inversion technique, Radio Sci., 41, RS4010, doi:10.1029/2005RS003355, 2006.

Rao, N. N.: Inversion of sweep-frequency sky-wave backscatter leading edge for quasiparabolic ionospheric layer parameters, Radio Sci., 9, 845-847, 1974.

Ruelle, N. and Landeau, T.: Interpretation of elevation-scan HF backscatter data from Losquet Island radar, J. Atmos. Sol. Terr. Phys., 56, 103-114, 1994.

Saillant, S., Auffray, G., and Dorey, P.: Exploitation of elevation angle control for a 2-D HF skywave radar, Proceedings of the Int. Conference on Radar, Australia IEEE Press, 662-666, 2003.
Su, F. F., Zhao, Z. Y., Deng, F., and Li, S. P.: Estimation of ground range on the sweep frequency backscatter leading edge, Adv. Space. Res., 47, 1187-1193, doi:10.1016/j.asr.2010.12.008, 2011.

Titheridge, J. E.: Ionogram Analysis with the Generalised Program POLAN, Report UAG-93, World Data Center A for SolarTerrestrial Physics, NOAA, E/GC2, Boulder, CO 80303, 1985.

Zhou, C., Zhao, Z. Y., Deng, F., Ni, B. B., and Chen, G.: Midlatitude ionospheric $\mathrm{HF}$ channel reciprocity: Evidence from the ionospheric oblique incidence sounding experiments, Radio Sci., 45, RS6017, doi:10.1029/2010RS004477, 2010. 